\title{
Traffic Safety Region Estimation Based on SFS- PCA-LSSVM: An Application to Highway Crash Risk Evaluation
}

\author{
Yanfang Yang \\ School of Traffic and Transportation \\ Beijing Jiaotong University \\ Beijing, 100044, China \\ yangyf@bjtu.edu.cn
}

\author{
Yong Qin \\ State Key Laboratory of Rail Traffic Control and Safety \\ Beijing Jiaotong University \\ Beijing, 100044, China \\ Corresponding author: yqin@bjtu.edu.cn
}

\begin{abstract}
Accurate real-time crash risk evaluation is essential for making prevention strategy in order to proactively improve traffic safety. Quite a number of models have been developed to evaluate traffic crash risk, by using real-time surveillance data. In this paper, the basic idea of traffic safety region is introduced into highway crash risk evaluation. Traffic safety region aims to describe the safe condition for highway, which means highway is under low risk of crash condition. Sequential forward selection (SFS), principal components analysis (PCA) and least squares support vector machine (LSSVM) are used comprehensively for traffic safety region estimation and classifying the traffic states (safe condition and unsafe condition). The method works by first extracting state variables from the observed traffic variables. Two statistics $T^{2}$ and SPE are calculated by SFS-PCA and used as the final state variables for traffic state space. Next, LSSVM is used to estimate the boundary of traffic safety region and identify the traffic states. To demonstrate the advantage of the proposed method, this study develops two crash risk evaluation models, namely SFS-LSSVM model and PCA-LSSVM model, based on crash data and non-crash data collected on freeway $I-880 \mathrm{~N}$ in Alameda. Validation results show that the method is of reasonably high accuracy for identifying traffic states.
\end{abstract}

Keywords - Traffic safety region, Crash risk evaluation, SFS, PCA, LSSVM

\section{INTRODUCTION}

Considerable effort has been devoted to shift from reactive (incident detection) to proactive (real-time crash risk prediction) traffic strategies in recent years, as the traffic safety continues to attract growing research interest [1]. Realtime crash risk evaluation models estimate the likelihood of crash occurrence over a short time period, such as $5 \mathrm{~min}$. The results have been utilized in traffic management system. Crash risk evaluation helps discern crash-prone conditions from normal conditions, which is essential for making prevention strategy. For example, the variable speed limit (VSL) system [2] is designed to determine appropriate traveling speed to reduce the crash risk, according the given current roadway and traffic conditions.

Existing traffic safety analysis can be classified into two categories [3]: aggregate analysis and disaggregate analysis.
Aggregate analysis assumes log-linear relationship [4] or nonlinear relationship $[5,6]$ between various variables and crash frequency, and then tries to estimate the crash frequency. Crash frequency analyses are traditional and feasible, however, more detail about each crash should be known. With the advanced traffic surveillance system, such as loop detector, speed radar, automatic identification system, traffic status prior to crash occurrence could be identified and matched with the corresponding crash. These matched data make disaggregate analysis be possible.

Disaggregate analysis focuses on estimating individual crash risk by using real-time traffic flow data from monitoring detectors. A considerable number of studies have developed regression models to establish a statistical relationship between the crash risk and traffic variables, such as simple/matchedcase logistic regression [7, 8], and Bayesian matched-case logistic regression [9]. It is found that traffic flow is significantly related to the risk of crash occurrence. However, a common assumption for regression models is that there is no dependency among the traffic variables, which could cause prediction models to be limited.

In order to overcome the limitation of regression models, data mining/machine learning approaches have been applied to crash risk evaluation. The common used methods include knearest neighbor models [10], neural networks [11], Bayesian network models [12,13] and support vector machines [14-16]. An accompanying issue to the approaches mentioned above is variable selection. Variable selection can help researchers identify and extract meaningful information, which may reduce the prediction running time and improve the prediction results. The widely used variable selection approaches include classification and regression tree [17], random forest [18], frequent pattern tree [19], etc.

Furthermore, some researchers applied reliability analysis of structural engineering to risk evaluation [20]. Reliability analysis is used to distinguish between safe and unsafe conditions by transforming the risk variables into a state space [21], which is the same with the risk evaluation's purpose. This study aims to use machine learning approaches to estimate the safe region of traffic system, termed traffic safety region, and 
identify the traffic conditions, i.e. safe state and unsafe state, from the viewpoint of region division. Firstly, state variables are extracted by combining sequential forward selection (SFS) and principal components analysis (PCA) from the observed traffic variables. Statistics $T^{2}$ and SPE, calculated from the observed traffic variables, are the state variables for the traffic state space. Then the boundary of the traffic safety region is calculated by two-class least squares support vector machines (LSSVM), and the identification of safe state and unsafe state for the traffic system is performed. The research results will promote a better understanding of the impact of traffic variables on the likelihood of crashes occurrence and the traffic safety region will help transportation professionals monitor the traffic system and develop effective crash prevention strategies on consequential crash events.

\section{METHODS}

\section{A. Safety Region Theory}

Safety region analysis and estimation theory was first applied to safety evaluation for power systems [22]. Zhang et. al. [23, 24] applied the idea of safety region into the monitoring and evaluation of the security state for rail system's key equipment. The results found that data mining/machine learning approaches performed well in safety region estimation and safety evaluation.

Supposing $X=\left\{X_{1}, X_{2}, \ldots, X_{m}\right\}$ is the observed traffic variable vector, $m$ is the number of observed traffic variables. Each $X_{i}$ is a column vector $\left[x_{1 i}, x_{2 i}, \ldots, x_{N i}\right]^{T}, i=1,2, \ldots, m$, and $N$ is the size of sample. State variables $F=\left\{f_{1}, f_{2}, \ldots, f_{k}\right\}=g(X)$ are extracted from $X$, where $g$ is a transform function, and $k \leq$ $m$. For a traffic system, the state space $U$ is constructed by state variables $F$ and consists of two sub regions (traffic safety region $E$ and traffic unsafe region $\bar{E}$ ), i.e., $U=E \cup \bar{E}$. Traffic safety region $E$ describes the normal (non-crash) operation area of traffic system. It means, if a state value of traffic system at time $t$, denoted by $P_{\mathrm{t}}$, belongs to the traffic safety region $E$, the traffic system is under the safe condition at time $t$. Otherwise, the status of traffic system is unsafe (i.e. under high risk of crash condition ). Fig. 1 illustrates a two dimensional state space. $F_{1}, F_{2}$ are two state variables and $P_{1}, P_{2}$ are two points which represent safe and unsafe states respectively. The main work of safety region estimation is to obtain the boundary, i.e. a classification and decision-making function to distinguish between safe and unsafe traffic states.

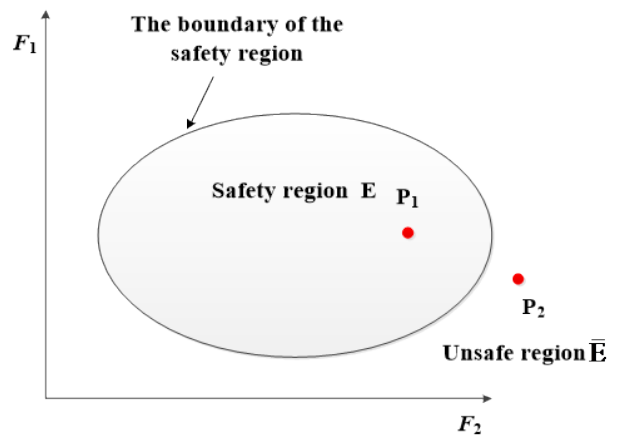

Fig. 1. Schematic diagram of safety region.

\section{B. SFS and PCA}

Many factors can contribute to the usefulness of data mining/machine learning algorithm in safety region estimation and crash risk evaluation. The quality of observed traffic variables (e.g. speed, volume, occupancy) is one of these factors. If the observed traffic variables contain irrelevant or redundant features, then the knowledge discovery process during the training becomes noisy and unreliable. In this paper, a state variable extraction method, combining SFS and PCA (SFS-PCA), is considered.

For SFS-PCA method, the input vector $V$ is composed of the observed traffic variable vector $X$ and the corresponding class label vector $Y . Y=\left\{Y_{1}, Y_{2}, \ldots, Y_{N}\right\}^{T}, Y_{l} \in\{1,-1\}$, where $l=1,2, \ldots, N, Y_{l}=1$ corresponds to crash case and $Y_{l}=-1$ corresponds to non-crash case.

The $V$ could be denoted as:

$$
\begin{aligned}
V & =\left\{\left(X_{l}, Y_{l}\right) \mid l=1,2, \ldots, N\right\} \\
& =\left\{\left[x_{l 1}, x_{l 2}, \ldots, x_{l m}, Y_{l}\right] \mid l=1,2, \ldots, N\right\}
\end{aligned}
$$

where $X_{l}$ is the lth sample in $X$, each $X_{l}$ contains $m$ observed traffic variables.

The goal of SFS-PCA method is to find a minimal set of state variables $F=\left\{f_{1}, f_{2}, \ldots, f_{k}\right\}(k \leq m$.) to represent the observed traffic variables in a lower dimensional state space. The SFS-PCA method could be described as follows:

The best possible subset $S$ of the observed traffic variables is selected by SFS firstly. SFS works in the opposite direction: starting from an empty set, $S$ is iteratively updated by including, in each step, the observe traffic variable vector $X_{i}$ which results in maximal score $G(S, X, M)$ [25-26]. Thus, the size of $S$, denoted by $d(d \leq m)$, is given by

$$
S_{d}=S_{d-1} \cup \arg \max _{X_{i}} G\left(S_{d-1} \cup X_{i}, X, M\right)
$$

where, $\mathbf{M}$ denotes the classification model applied in the task. In this paper, k-nearest neighbor model is used as the classification model.

After the SFS procedure, the final state variable set $F$ is extracted from $S$ by PCA. PCA decomposes $S$ into two subspaces (i.e., a lower dimensional feature subspace composed of principle components and a residual subspace) by multiple projections [27]. Two statistic indicators, $T^{2}$ and squared prediction error (SPE), are calculated in the two subspaces respectively. $T^{2}$ could reflect the change of the principle component model in feature subspace and SPE could measure the interference and noise in the residual subspace [28].

$T^{2}$ and SPE can be calculated using the following equations respectively:

$$
\begin{aligned}
& T_{l}^{2}=S_{l} P_{b} \lambda^{-1} P_{b}^{T} s_{l}^{T}, \quad l=1,2, . ., N \\
& S P E_{l}=s_{l}\left(I-P_{b} P_{b}^{T}\right) s_{l}^{T}, \quad l=1,2, . ., N
\end{aligned}
$$

where, $s_{l}$ is the lth sample in subset $S, P_{b}$ is the matrix of the $b$ loading vectors, which could be calculated by PCA, $I$ is the identity matrix. 


\section{LSSVM}

LSSVM classifier is one particular sample of support vector machine (SVM). LSSVM classifier maps the input vectors into a high dimensional feature, then finds an optimal separating hyper plane by using maximum Euclidean distance to the nearest point $[29,30]$.

Given a state variable set $\left\{\left(F_{l}, O_{l}\right) \mid l=1,2, \ldots, N\right\}$ where $F_{l}$ is the input data, i.e. the state variables mentioned above, and $O_{l}$ is the output data, i.e., the classification results. To classify the state variable set, LSSVM has to find the optimal (with maximum margin) separating hyper plane, which could be formulated in the following form:

$$
\left\{\begin{array}{l}
\min J(\omega, \xi)=\frac{1}{2} \omega^{T} \omega+\frac{1}{2} \gamma \sum_{l=1}^{N} \xi_{l}^{2} \\
\text { s.t. } O_{l}\left[\omega^{T} \varphi\left(F_{l}\right)+\eta\right]=1-\xi_{l}, l=1,2, \ldots, N
\end{array}\right.
$$

where $J$ is an objective function, $\omega$ is the normal vector of the separating hyper plane, $\eta$ is the corresponding bias term and $\varphi\left(F_{l}\right)$ is the nonlinear mapping function ,which projects the state variable $F_{l}$ into a high-dimensional space. $\gamma$ is the regularization parameter.

The corresponding Lagrange function is:

$$
\left.L(\omega, \eta, \xi, \alpha)=J(\omega, \xi)-\sum_{l=1}^{N} \alpha_{l}\left\{O_{l}\left[\omega^{T} \varphi\left(F_{l}\right)+\eta\right]\right\}-1+\xi_{l}\right\}
$$

where $\alpha_{l}$ is a Lagrange multiplier. According to the conditions for optimality yield, the following equations must be satisfied: $\partial L / \partial \omega=0, \partial L / \partial \eta=0, \partial L / \partial \xi_{l}=0$, and $\partial L / \partial \alpha_{l}=0$, which could be simplified by $\sum_{l=1}^{N} \alpha_{l} \varphi\left(F_{l}\right)$ $=\omega, \sum_{l=1}^{N} \alpha_{l}=0, \alpha_{l}=\gamma \xi_{l}$, and $O_{l}\left[\omega^{T} \varphi\left(F_{l}\right)+\eta\right]+\xi_{l}-1=0$ $(l=1,2, \ldots, N)$, respectively.

Eliminate $\omega$ and $\xi$, obtain linear equations as follows:

$$
\left[\begin{array}{cc}
0 & \mathbf{1}^{T} \\
\mathbf{1} & \mathbf{K}+\gamma^{-1} \mathbf{I}
\end{array}\right]\left[\begin{array}{l}
\eta \\
\boldsymbol{\alpha}
\end{array}\right]=\left[\begin{array}{l}
0 \\
\mathbf{O}
\end{array}\right]
$$

where $\mathbf{1}=[1,1, \ldots, 1]^{\mathrm{T}}, \mathbf{O}=\left[O_{1}, O_{2}, \ldots, O_{N}\right]^{\mathrm{T}}, \boldsymbol{\alpha}=\left[\alpha_{1}, \alpha_{2}, \ldots\right.$, $\left.\alpha_{\mathrm{N}}\right]^{\mathrm{T}}$. I is an identify matrix and $\mathbf{K}$ is the kernel matrix. With $\eta$ and $\boldsymbol{\alpha}$ known, the classification decision-making function is

$$
f\left(F_{l}\right)=\operatorname{sgn}\left[\sum_{l=1}^{N} \alpha_{l} K\left(F_{l}, F\right)+\eta\right]
$$

In this study, the radial basis function (RBF) is selected as the kernel function and given as follows:

$$
K\left(F_{l}, F\right)=\exp \left(\frac{-\left\|F-F_{l}\right\|^{2}}{2 \sigma^{2}}\right)
$$

where, $\sigma$ is the width of the RBF kernel.

\section{Implementation Procedures for SFS-PCA-LSSVM Method}

The implementation procedures for traffic safety region estimation and crash risk evaluation based on SFS- PCALSSVM are shown as follows:

Step 1. Collect crash data and non-crash data as the training data for SFS - PCA - LSSVM method. Crash data include crash information (e.g. time, location) and the matched traffic flow data collected from the traffic surveillance system (e.g. speed, volume, occupancy). Non-crash data are traffic flow data in a given time interval when the traffic is under safe condition.

Step 2. Obtain subset $S$ from the observed traffic variable set by using SFS.

Step 3. Process subset $S$ of the observed traffic variables by PCA, and calculate statistics $T^{2}$ and SPE. The two statistics form a two-dimensional statistical feature vector for each sample, and the feature vectors would be the final state variable set $F$.

Step 4. Use the two-dimensional statistical feature vectors as the input data for LSSVM. Classify the traffic states into safe state or unsafe state and obtain the best classified curve which is the boundary of the traffic safety region.

\section{DATA PREPARATION}

In this paper, crash data and traffic flow data were collected from a 35-mile freeway section from milepost 10.55 to milepost 45.42 on the I-880 freeway in Alameda in the United States. A total of 70 loop detector stations were located along the selected direction (northbound) of the freeway segment. The average spacing between detector stations was approximate 0.5 mile. Crash data and the paired real-time traffic flow data were collected from January 1, 2011 to December 31, 2012. A total of 417 crashes were identified and used for further data analysis.

Traffic data were collected from the highway Performance Measurement System (PeMS) which was computerized database maintained by the California Department of Transportation (Caltrans) [31]. The 30-s raw loop data, i.e. speed, volume and occupancy, for each lane were collected from the Caltrans PeMS database. The raw data further aggregated to 5-min intervals. Each crash was assigned to the nearest loop detector (as shown in Fig. 2), and the traffic data in the time interval between 5 and 10 min prior the crash time were selected to represent the traffic condition [14]. At the same time, the traffic data of upstream and downstream were also extracted. For example, if a crash happened at 13:32, at the milepost 15.46. Traffic condition of nearest loop detector at milepost 15.54 in time intervals 13: 20 and 13:25 was the corresponding traffic status for this crash. To eliminate the geometric characteristics' influences on crash risk evaluation [14], matched case-control structure was used to extract noncrash data. For each specific crash case, two non-crash cases, one week before and one week after the crash time, were identified and matched. For example, a crash happened on April 26, 2011, the corresponding non-crash cases (April 19, 2011 and May 3, 2011) at the location of crash occurrence were selected. In this study, a total of 837 non-crash cases 
were identified.

For each sample, average and standard deviation values of the speed, occupancy, and volume for the three detectors $(2 \times 3 \times 3=18$ variables) constitute the observed traffic variable set for the traffic safety region estimation and crash risk evaluation.

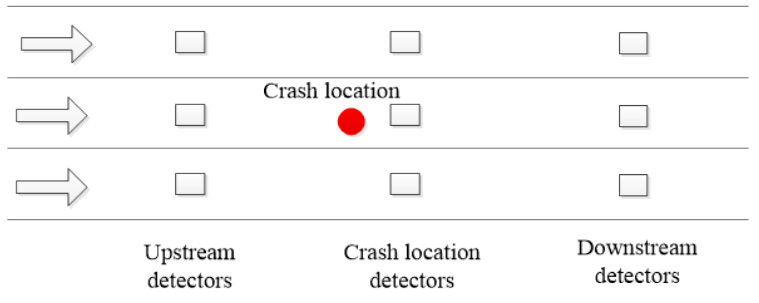

Fig. 2. Illustration of field data collection.

\section{Modeling Results AND Discussion}

\section{A. State Variable Extraction Using SFS-PCA}

Variables important scores are calculated via SFS and the subset $S$ is determined based on the scores. In this study, 8 observed traffic variables are selected from the 18 observed traffic variables, i.e., downstream standard deviation of speed (DDS), crash location average occupancy (CAO), upstream standard deviation of speed (UDS), crash location standard deviation of occupancy (CDO), downstream average speed (DAS), upstream standard deviation of occupancy (UDO), crash location standard deviation of speed (CDS), and downstream average occupancy (DAO). Furthermore, multicollinearity test for the 8 observed traffic variables has been carried out by using SPSS and the correlation coefficients between two variables in the subset are calculated, as listed in Table 1 . The results imply that some of variables exist highly correlated relation, e.g. the correlation between DAO and DAS is 0.825 , approximating to 1 , which suggests that a further analysis should be conducted on the selected observed traffic variables before being used in the classification model.

TABLE I. CORRELATION MATRIX FOR SELECTED OBSERVED TRAFFIC VARIABLES

\begin{tabular}{|c|c|c|c|c|c|c|c|c|}
\hline & DDS & CAO & UDS & CDO & DAS & UDO & CDS & DAO \\
\hline DDS & 1 & 0.069 & -0.014 & 0.057 & 0.171 & 0 & -0.413 & 0.042 \\
\hline CAO & 0.069 & 1 & 0.096 & -0.357 & 0.041 & -0.202 & 0.153 & $\begin{array}{c}- \\
0.348\end{array}$ \\
\hline UDS & -0.014 & 0.096 & 1 & 0.237 & 0.045 & -0.729 & -0.422 & 0 \\
\hline CDO & 0.057 & -0.357 & 0.237 & 1 & 0.097 & -0.312 & -0.699 & 0.121 \\
\hline DAS & 0.171 & 0.041 & 0.045 & 0.097 & 1 & 0.016 & -0.115 & 0.825 \\
\hline UDO & 0 & -0.202 & -0.729 & -0.312 & 0.016 & 1 & 0.283 & 0.053 \\
\hline CDS & -0.413 & 0.153 & -0.422 & -0.699 & $\begin{array}{c}- \\
0.115\end{array}$ & 0.283 & 1 & $\begin{array}{c}- \\
0.096\end{array}$ \\
\hline DAO & 0.042 & -0.348 & 0 & 0.121 & 0.825 & 0.053 & -0.096 & 1 \\
\hline
\end{tabular}

In order to eliminate the high correlation among the selected observed traffic variables, the PCA is applied to the observed traffic variable subset. Cumulative percentage of total variation $80 \%$ rule is used to determine the number of components. Finally, three components are chosen. Fig. 3 shows the cumulative proportion for the first 3 components. The corresponding two statistics $T^{2}$ and SPE are calculated using (3) and (4), which would be the final input state variable set to the LSSVM method.

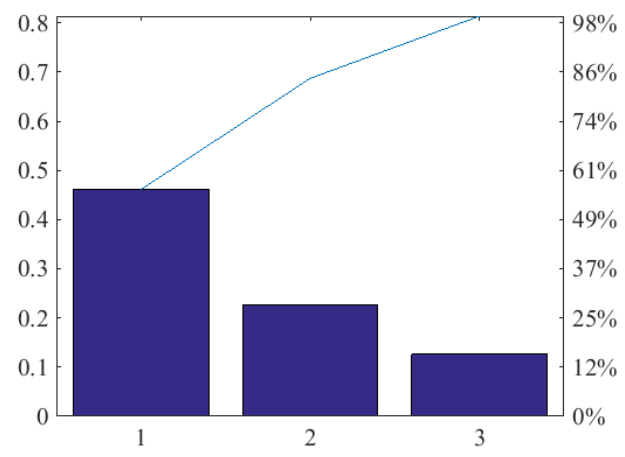

Fig. 3. The cumulative proportion of the first 3 components.

\section{B. Model Results}

In this section, the performance evaluation methods used to evaluate the proposed method are presented. The experimental results are given and the observations based on these results are discussed.

The $k$-fold cross-validation is applied during the classification experiments. The dataset is divided into $k$ subsets, and the holdout method is repeated $k$ times. Each time, one of the $k$ subsets is used as the test set and the other $k-1$ subsets are put together to form the training set. In this study, the 2 -fold cross validation is taken, i.e. $50 \%$ of the whole data set is used as the training dataset and the rest $50 \%$ of the whole data is used as the validation dataset. The classification result is shown in Fig. 4. The left column is the classification result of training dataset, and the traffic safety region boundary is also estimated. The right column plots the testing data points on the classified region. In Fig. 4, it is intuitive that the SFS-PCA-LSSVM method works efficiently in classifying the traffic statuses. However it is necessary to evaluate these results in a quantitative way.

The classification accuracy for the dataset is measured by correct rate (CR) according to

\section{$\mathrm{CR}=\frac{\text { the total number of samples correctly classified }}{\text { the total number of samples }} \times 100 \%$}

TABLE II. CR FOR THREE METHODS IN DifFERENT TRAINING DATASET

\begin{tabular}{cccc}
\hline $\begin{array}{c}\text { Training } \\
\text { dataset }\end{array}$ & $\begin{array}{c}\text { SFS- } \\
\text { LSSVM }\end{array}$ & $\begin{array}{c}\text { PCA- } \\
\text { LSSVM }\end{array}$ & $\begin{array}{c}\text { SFS-PCA- } \\
\text { LSSVM }\end{array}$ \\
\hline 2-fold & $75.54 \%$ & $67.70 \%$ & $88.16 \%$ \\
3-fold & $76.12 \%$ & $68.34 \%$ & $88.28 \%$ \\
4-fold & $77.19 \%$ & $68.82 \%$ & $88.84 \%$ \\
\hline
\end{tabular}




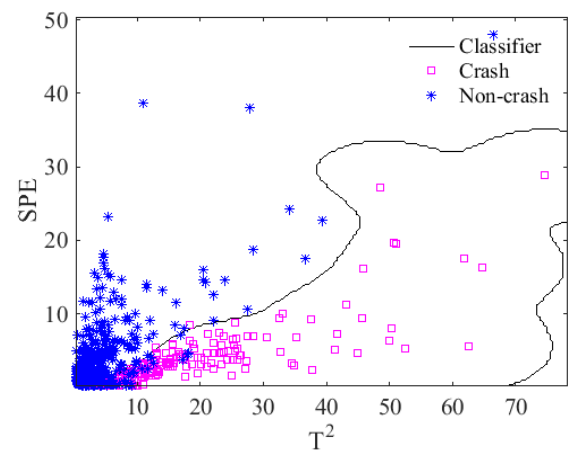

(a)

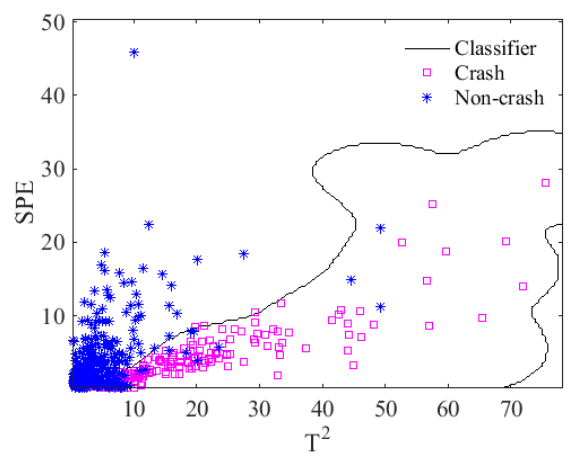

(b)

Fig. 4. 2-Fold classification results: (a) training dataset (b) test dataset.

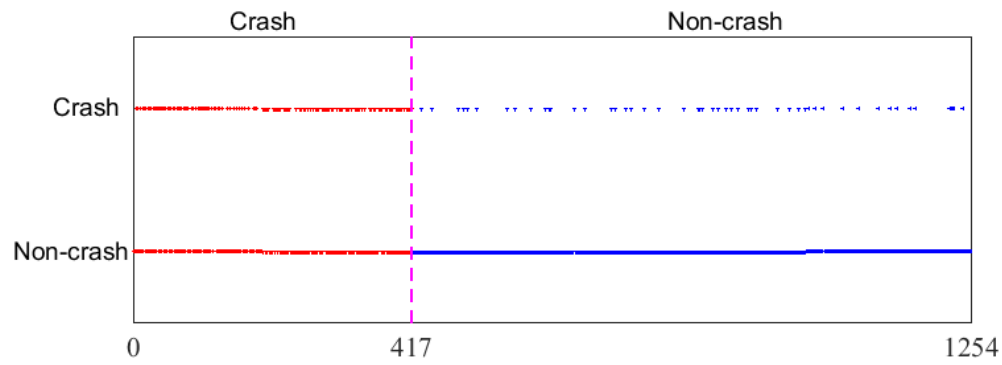

(a)
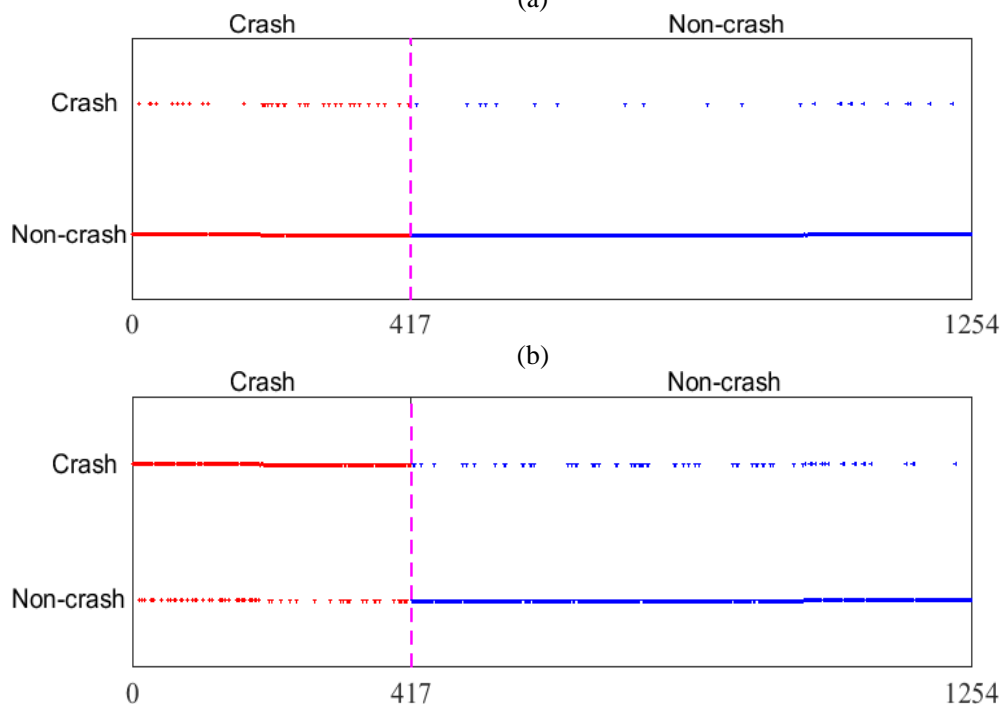

(c)

Fig. 5. The classification results of three methods: (a) SFS-LSSVM (b) PCA-LSSVM (c) SFS-PCA-LSSVM

The classification performance of the proposed method was compared with the SFS-LSSVM and PCA-LSSVM methods as listed in the 1st row of the Table 2. Furthermore, in order to demonstrate the classification performance, the classification results of three methods are plotted. As shown in Fig. 5, the horizontal axis represents the number of state points. The 1st 417th points are crash state samples, the 418th $\sim 1254$ th are non-crash state points. The whole area is divided into two subareas by pink dotted lines. Vertical axis from bottom to top represents the two classes of data samples. It can conclude that the proposed method performs better than other two methods.

The traffic safety region estimation is dependent on the size of training dataset. In this study, two additional crossvalidation experiments are conducted, i.e. 3-fold crossvalidation and 4-fold cross-validation. The CR values for the three models mentioned above are calculated. As listed in Table 2, all the CR values of SFS-PCA-LSSVM are higher than that of other two models. While the size of training dataset becomes bigger, the corresponding CR values increase. It can be conclude that for different size of 
training dataset, sufficient training data may improve the classification results.

\section{Conclusions}

This paper presents an intelligent method based on SFSPCA-LSSVM for traffic safety region estimation and traffic sate classification. The traffic condition data, including crash data and non-crash data, is obtained and the dimension of these traffic variables is 18. SFS method is used and selects 8 observed variables as the sub variable set, which could be found that some of observed variables exist high correlation. In order to reduce the high correlation among selected variables, the PCA is applied to extract state variables from the variable subset. Two statistics, i.e. $T^{2}$ and SPE, are calculated and given to LSSVM classifier in classification stage of SFS-PCA-LSSVM traffic safety region estimation system. Final experimental results show that the classification accuracy rates of the proposed method are all reasonable high accuracy, i.e. all above $88 \%$, and SFS-PCA-LSSVM method outperforms two given methods, SFS- LSSVM and PCA-LSSVM methods.

\section{ACKNOWLEDGMENT}

This research was supported by the National Science \& Technology Support Program of China (Grant No. 2014BA G01B02), and Beijing Municipal Institute of Science and Technology (Grant No. PXM2015_178215_000008).

\section{REFERENCES}

[1]. C. Shew, A. Pande, and C. Nuworsoo. "Transferability and robustness of real-time freeway crash risk assessment”. Journal of Safety Research, Vol. 46 , pp. 83-90, 2013

[2]. S. Pan, L. Jia, N. Zou, and S. Park. "Design and implement of a variable speed limit system with travel time display - a case study in Maryland,” USA. In 17th ITS World Congress, Busan 2010

[3]. Q. Shi, and M. Abdel-Aty. "Big data application in real-time traffic operation and safety monitoring and improvement on urban expressways," Transportation Research Part C: Emerging Technologies. 2015 in press

[4]. F. Guo, X. Wang, and M. Abdel-Aty. "Modeling signalized intersection safety with corridor-level spatial correlation,” Accident Analysis and Prevention, Vol.42, pp. 84-92, 2010

[5]. R. Yu, and M. Abdel-Aty. "Investigation the different characteristics of weekday and weekend crashes,” Journal of Safety Research, Vol. 46, pp. 91-97, 2013

[6]. C. Wang, M. A. Quddus, and S. G. Ison. "Impact of traffic congestion on road accidents: a spatial analysis of the M25 motorway in England," Accident Analysis and Prevention, Vol. 41, No.4, pp.798-808, 2009

[7]. M. Abdel-Aty, N. Uddin, F. Abdala, A. Pande, and L. Hsia. "Predicting freeway crashes based on loop detector data using mathed case-control logistic regression,” Transportation Research Record: Journal of Transportation Research Board, No.1897, Transportation Research Board of the National Academies, Washington, D.C. , pp. 88-95, 2004

[8]. Z. Zheng, S. Ahna, and C. "Monsere. Impact of traffic oscillations on freeway crash occurrences," Accident Analysis and Prevention, Vol. 42 , pp. 626-636, 2010

[9]. M. Abdel-Aty, H. M. Hssan, M. Ahmed, and A. S. Al-Ghamdi. "Real-time prediction of visibility related crashes," Transportation Research Part C: Emerging Technologies, Vol. 24, pp.288-298, 2012

[10]. Y. Lv, S. Tang, and H. Zhao. "Real-time highway traffic accident prediction based on the $\mathrm{k}$-mearest neighbor method,” International Conference on Measuring Technology and Mechatronics
Automation, Vol. 3, pp. 547-550, 2009

[11]. M. Abdel-Aty, A. Pande, A. Das, and W. J. Knibbe. "Assessing safety on Dutch freeway with data from infrastructure-based intelligent transportation systems,” Transportation Research Record: Journal of Transportation Research Board, No. 2083, Transportation Research Board of the National Academies, Washington, D.C., pp. 153-161, 2008

[12]. O. H. Kwon, W. Rhee, and Y. Yoon. "Application of classification algorithm for analysis of road safety risk factor dependencies," Accident Analysis and Prevention, Vol. 75, pp. 1-15, 2015

[13]. C. Xu, P. Liu. W. Wang, and Z. Li. "Identification of freeway crashprone traffic conditions for traffic flow at different levels of service,” Transportation Research Part A: Policy and Practice, Vol. 69 , pp. 58-70, 2014

[14]. R. Yu, and M. Abdel-Aty. "Utilizing support vector machine in real-time crash risk evaluation,” Accident Analysis and Prevention, Vol.51, pp. 252-259, 2013

[15]. R. Yu, and M. Abdel-Aty. "Analyzing crash injury severity for mountainous freeway incorporating real-time traffic and weather data,” Safety Science, Vol. 63, pp. 50-56, 2014

[16]. R. Gang, and Z. Zhou. "Traffic safety forecasting method by particle swarm optimization and support vector machine,” Expert Systems with Applicaton, Vol.38, pp.10420-10424, 2011

[17]. M. Hosain, and Y. Muromachi. "Understanding crash mechanism on urban expressways using high-resolution traffic data," Accident Analysis and Prevention, Vol.57, pp. 17-29, 2013

[18]. M. M. Ahmed, and M. Abdel-Aty. "The viability of using automatic vehicle identification data for real-time crash prediction," IEEE. Transactions on Intelligent Transportation System, Vol. 13, pp.459468, 2012

[19]. L. Lin, Q. Wang, and A. W. Sadek. "A novel variable selection method based on frequent pattern tree for real-time traffic accident risk prediction," Transportation Research Part C: : Emerging Technologies, Vol.55, pp. 444-459, 2015

[20]. R. Yu, Q. Shi, and M. Abdel-Aty. "Feasibility of incorporating reliability analysis in traffic safety investigation," Transportation Research Record: Journal of Transportation Research Board, No. 2386, Transportation Research Board of the National Academies, Washington, D.C., pp. 35-41, 2013

[21]. A. S. Nowak , and K.R. Collins. Reliability of Structures. CRC Press, 2012

[22]. F. Wu, and S. Kumagai. "Steady-state security regions of power systems," IEEE Transaction on Circuits and Systems, Vol. 29, pp. 703-711, 1982

[23]. Y. Zhang, Y. Qin, Z. Xing, L. Jia, and X. Cheng. "Roller bearing safety region estimation and state identification based on LMDPCA-LSSVM,” Measurement, Vol. 46, pp. 1315-132, 2013

[24]. Y. Zhang, Y. Qin, Z. Xing, L. Jia, and X. Cheng. "Safety region estimation and state identification of rolling bearing based on statistical feature extraction," Shock and Vibration, Vol. 20, pp.833846, 2013

[25]. Y. Liu, and Y. F. Zheng. "FS_SFS: A novel feature selection method for support vector machine,” Pattern Recognition, Vol.39, pp.1333145, 2006

[26]. J. Pohjalainen, O. Räsänen, and S. Kadioglu. "Feature selection methods and their combinations in high-dimensional classification of speaker likability, intelligibility and personality traits," Computer Speech and Language, Vol.29, pp.145-171, 2015

[27]. K. Pearson. "On lines and planes of closest fit to systems in space," Phisophical Magazine, Vol. 2, pp. 559-573, 1901

[28]. A. J.Richard and W. W. Dean. Applied multivariate statistical analysis. Six ed., Prentice Hall, New Jersey, 2007

[29]. D. Çalişir, and E. Dogantekin. "A new intelligen hepatitis diagnosis system: PCA- LSSVM,” Expert Systems with Application, Vol. 38, pp.0705-10708, 2011

[30]. H. Xu, and G. Chen. "An intelligent fault identification method of rolling bearing based on LSSVM optimized by improved PSO," Mechanical Systems and Signal Processing, Vol.35, pp. 167-175, 2013

[31]. C. Xu, P. Liu, W., Wang, and Z. Li. "Evaluation of the impacts of traffic states on crash risk on freeways," Accident Analysis and Prevention, Vol. 47, pp. 162-171, 2012 